ment à l'hectare, au moins n'aurons-nous pas, cette année, à en juger des premières pêches qui sont, au point de vue sanitaire, absolument satisfaisantes, à redouter que les produits de nos étangs soient, au même degré que les deux années précédentes, infestés de poux, de Gyrodactylus, de gale, etc., dont le développement, à n'en pas douter, est dù en grande partie à une eau stagnante, insuffisamment renouvelée par suite de la sécheresse.

Vous me direz que quelques Argules sur la queue d'un Poisson et des Sangsues en petites quantités ne sont généralement pas un obstacle à la vente; c'est entendu, encore qüil convienne de ne pas s'en recommander spécialement.

Mais il faut avoir éprouvé les déboires inattendus d'une pèche de Cyprinidés atteints de variole (que nous appelons vulgairement gale, masse grélatineuse ou sorte de lait caillé durci, adhérenl fermement aux nageoires et aux écailles plus spécialement de la (Carpe) pour savoir combien sont désagréables à la vue les sujets atleints, et à quel point en est embarrassé le pisciculteur non averti, dont la récolte, sinon sans valeur, est du moins inutilisable avant complète guérison.

Voyez, au contraire, le Poisson cette année. II est beau, gras, dodu et d'une présentation impeccable.

Ni nous, ni le Gouvernement, ne pouvons rien aux conditions atmosphériques, mais quitte à subir quelques moins-values sur la production, n'avons-nous bien des raisons de ne pas maudire cette année pluvieuse si nous pouvons lui attribuer la cause d'une régression sensible de la vermine sur nos Poissons?

\title{
L'AVENIR DE LA CARPE
}

\section{Par M. Guy RIORGES}

A notre époque, les conditions économiques se transforment rapidement.

En ce qui concerne la Carpe, le marché allemand, encombré, nous manque de plus en plus ; il nous faut trouver de nouveaux débouchés et, surtout, créer une consommation en France.

Mais notre marché intéricur n'est pas organisé. La vente aux Halles, entourée de garanties théoriques, n'est, en fait, qu'une déception pour le producteur et pour le consommateur; nous reviendrons sur cette question. Le gros public veut de la Carpe-portion de I à 2 livres qui ne peut être un mets assez fin pour s'imposer et conquérir une consommation progressive. La bonne Carpe de 3 livres et plus n'est pas connue, pas appréciée à sa valcur, pas demandée : le souvenir de la petite Carpe lui nuit. Elle représente aussi une dépense trop forte pour li masse des consommateurs.

Or, un fait nouveau se produit : la pèche maritime utilise des frigori- 
fiques perfectionnés qui permetlent la capture de dizaines de tonnes de Poissons par coup de chalut, loin des còtes. Se Poisson de mer va encore baisser de prix. Seul le Poisson de luxe grardera des cours élevés.

La larpe d'une live, qui est ou maigre ou trop jeune, tombera à des cours évidemment inférieurs au prix de revient. Surproduite ou non, elle ne payera plus, si tant est que!le ait jamais pay.

Reste la belle Carpe de 3 à 6 livres et de 3 à $\{$ étés, qui vaut le Turbot ill point de vue gastronomique. Si elle reste un Poisson peu goûté, elle disparaitra parce quelle se vendra en dessons de son prix de revient. Il ny a donc de salut pour la Carpe que dans sa transformation en Poisson de luxe. De fait, elle en est un (I). Mais comment lui trouver des consommateurs nombreux ?

En la faisant bonne, done grosse : et abordable aux petits ménages....

Il n'y a pas si longlemps que la propagrande marilime a réussi à faire accepter par le public les gros Poissons décapités, vidés, débités en poriions. Il fallait y arriver ou ne plus vendre les grands Poissons.

La mème nécessité imposera la mème solution pour la Carpe, ou bien la Carpe disparaîtra du marché.

Nous n'aurons des consommateurs qu'aver de la Carpe bonne à manger, done grosse. Nous ne vendrons la grosse Carpe que débitée en tranches, ce qui se fait en Pologrne, en lllemagne, très couramment. C'est une affaire de mode, d'habitude.

Le frigo entrant dans les morurs, il n'y aura bientôt plus d'intérèt à rendre vivante la Carpe, surtout si le zroùl du public préfère la belle tranche charnue à la carpille arêteuse ou mollasse. 11 y aura donc, à la place des viviers, des frigo. Conservation, manutention, transports coûteront moins cher : le prix de revient s'abaissera d'environ $5 \%$, et la perte de poids, le risque de mortes non vendues, dimimeront de nis \%. Je prix de la Carpe ne sera plus obligé de faire la culbute entre la chaussée et le premier revendeur. Nous pourrons avoir des entrepots de Coopératives it régler le marché.

Mais la petile carpille, mème hénéficiant (?) du frigo, se vendra-t-elle alors à un prix rémunérateur? Elle auma perdu sa seule qualité, la vie sur l'étal et, vivante, elle ne pourra se vendre assez cher, ì côté du poisson de mer de meme rlasse ganstronomique, surabondant.

Il est done probable que surviva seule la carpe de plusieurs lives, vendue vivante comme luxe, ou frigrorifíe et découpée comme le bon Poisson de mer.

Il n'est pas trop trit pour l'envisager et lui préparer son marché (2).

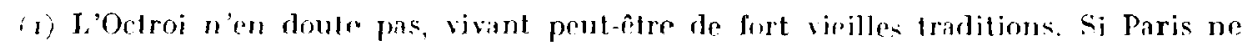
foit paver que 2 c centimes, Boulogne el Levallois taxent la Carpe à 9 sous, Saintlienne a mo sous !

(a) In essai de decoupage, tente par les magasins dalimentation du Casino, a Saintitienne, a róussi récemment. 